\title{
GIS-based hillshade representation combined with relative relief as pseudo shade to enhance micro-topography
}

\author{
Keiko Suzuki $^{\text {a, *, Go Ishikawa }}{ }^{\mathrm{b}}$ \\ ${ }^{a}$ Tokyo Map Research Inc. Tokyo, Japan.k-suzuki@t-map.co.jp \\ ${ }^{b}$ Tokyo Map Research Inc. Tokyo, Japan. ishikawa@t-map.co.jp \\ * Corresponding author
}

Keywords: Hillshade, Light sources, Relative relief, DEM, GIS

\begin{abstract}
:
Hillshade is a useful terrain representation that can express complicated topographic features on a map in 3D. However, the visual effects depend on the azimuth and the altitude of the light source corresponding to the target area. The purpose of this study is to provide a new method of GIS-based hillshade which can recognize various topographies in greater detail while reducing the dependency on the light source.
\end{abstract}

The azimuth dependency of the light source can be traced back to the time of handwritten mapping, when cartographers combined multiple hillshades with differing azimuth settings. The authors of this study first tried to combine three types of hillshade (A, B and C, Figure1-3) that had not only different light sources but also different grayscale gradients. Hillshade A had a light source from the upper side of the map with the darkest grayscale gradient. Hillshade B had a light source from the left side with a medium grayscale gradient. Finally, Hillshade C's light source was from the right side of the map with the brightest grayscale gradient. We confirmed that combining Hillshades A to $\mathrm{C}$ reproduces the same natural shading as that of a single light source from the upper left.

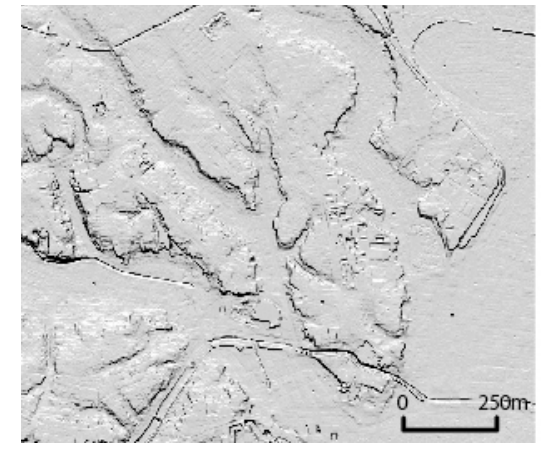

Figure 1. Hillshade A

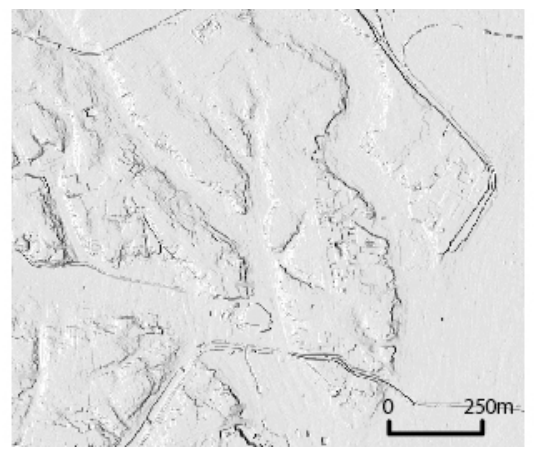

Figure 2. Hillshade B

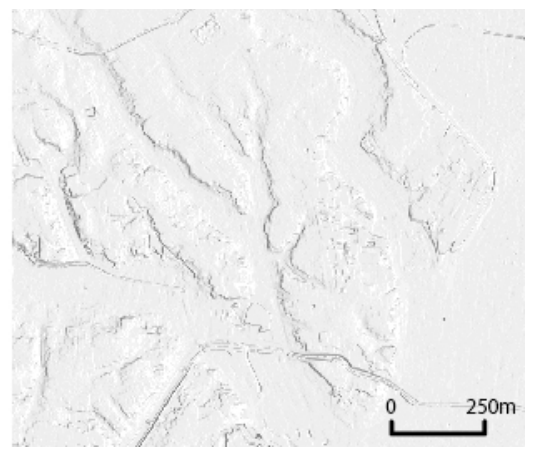

Figure 3. Hillshade C

The next purpose of the study was to enhance the topographic features of hillshade maps without overemphasizing the elevation factor in the GIS. We attempted to combine various topographic values through spatial analysis using a DEM in the GIS. Thus, we decided to combine a relative relief - a topographic value of maximum elevation change rate-as a pseudo shade (Figure4). However, because synthesizing the amount of relative relief in all directions overemphasizes the topography, we applied this only to downslope surfaces on the map created in the GIS. This pseudo shade works better for emphasizing micro-topography than does an upper light source in Hillshade A (Figure5).

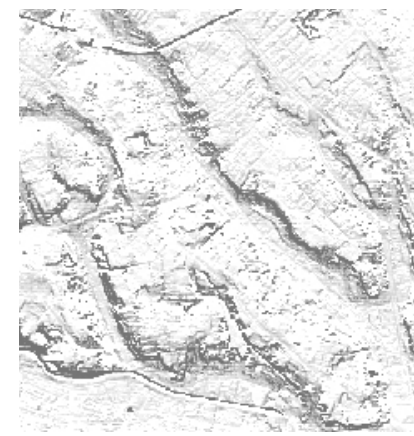

Figure 4. Relative relief

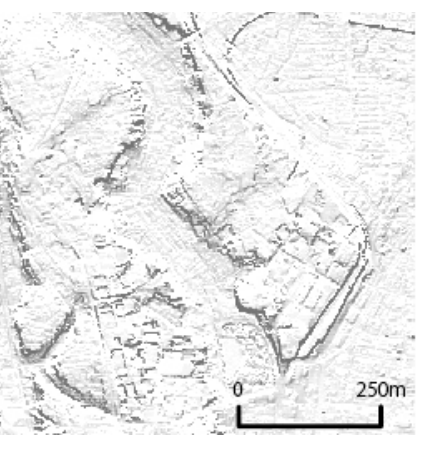

Figure 5. New hillshade combined with pseudo shade

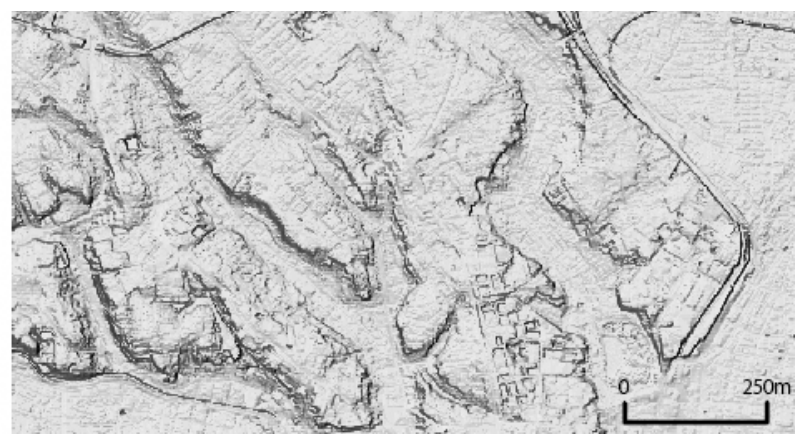

